\title{
Orbital Parameters of the PSR B1620-26 Triple System
}

\section{Z. Arzoumanian}

Department of Astronomy, Cornell University

K. Joshi and F. A. Rasio

Department of Physics, Massachusetts Institute of Technology

S. E. Thorsett

\section{Department of Physics, Princeton University}

\begin{abstract}
Previous timing data for PSR B1620-26 were consistent with a second companion mass $m_{2}$ anywhere in the range $\sim 10^{-3}-$ $1 M_{\odot}$, i.e., from a Jupiter-type planet to a star. We present the latest timing parameters for the system, including a significant change in the projected semi-major axis of the inner binary, a marginal detection of the fourth time derivative of the pulse frequency, and the pulsar proper motion (which is in agreement with published values for the proper motion of M4), and use them to further constrain the mass $m_{2}$ and the orbital parameters. Using the observed value of $\dddot{f}$, we obtain a one-parameter family of solutions, all with $m_{2} \lesssim 10^{-2} M_{\odot}$, i.e., excluding stellar masses. Varying $\dddot{f}$ within its formal $1 \sigma$ error bar does not affect the mass range significantly. However, if we vary $\dddot{f}$ within a $4 \sigma$ error bar, we find that stellar-mass solutions are still possible. We also calculate the predicted rate of change of the projected semi-major axis of the inner binary and show that it agrees with the measured value.
\end{abstract}

\section{Introduction}

The millisecond radio pulsar PSR B1620-26, in the globular cluster M4, has a low-mass binary companion (probably a white dwarf of mass $m_{1} \approx 0.3 M_{\odot}$ for a pulsar mass $m_{p}=1.35 M_{\odot}$ ) in a 191 day low-eccentricity orbit. In addition, its unusually large frequency second and third derivatives indicate the presence of a second companion in a wider orbit around the inner binary (Backer et al. 1993; Thorsett et al. 1993; Michel 1994). Such a hierarchical triple configuration is expected to be produced quite easily in a dense globular cluster through dynamical interactions between binaries (Rasio et al. 1995; Sigurdsson 1995). 


\section{Latest Timing Data}

\begin{tabular}{ll}
\hline Right ascension (J2000.0) & $16^{\mathrm{h}} 23^{\mathrm{m}} 38^{\mathrm{s}} 2228(6)$ \\
Declination (J2000.0) & $-26^{\circ} 31^{\prime} 53^{\prime \prime} 74(4)$ \\
Proper motion RA $\left(\mathrm{mas} \mathrm{yr}^{-1}\right)$ & $-16(3)$ \\
Proper motion Dec $\left(\mathrm{mas} \mathrm{yr}^{-1}\right)$ & $-30(18)$ \\
Dispersion measure $\left(\mathrm{cm}^{-3} \mathrm{pc}\right)$ & $62.8627(8)$ \\
& \\
Spin period $P(\mathrm{~ms})$ & $11.075750892214(6)$ \\
Spin frequency $f(\mathrm{~Hz})$ & $90.2873321847(5)$ \\
$\dot{f}\left(\mathrm{~s}^{-2}\right)$ & $-6.065(2) \times 10^{-15}$ \\
$\ddot{f}\left(\mathrm{~s}^{-3}\right)$ & $1.897(4) \times 10^{-23}$ \\
$\ddot{f}\left(\mathrm{~s}^{-4}\right)$ & $1.2(2) \times 10^{-32}$ \\
$\dddot{f}\left(\mathrm{~s}^{-5}\right)$ & $-1.7(5) \times 10^{-40}$ \\
Epoch of $f(\mathrm{MJD})$ & 48365.0 \\
& \\
Projected semi-major axis $x(\mathrm{~s})$ & $64.809478(8)$ \\
Orbital period $P_{b}(\mathrm{~s})$ & $16540653(6)$ \\
Eccentricity $e$ & $0.0253151(3)$ \\
Time of periastron $T_{0}\left(\mathrm{MJD}^{-4}\right)$ & $48345.3771(3)$ \\
Angle of periastron $\omega$ & $117.1296(6)$ \\
Mass function $\left(M_{\odot}\right)$ & $7.975 \times 10^{-3}$ \\
& \\
Advance of periastron $\dot{\omega}\left({ }^{\circ} \mathrm{yr}{ }^{-1}\right)$ & $(-2.9 \pm 2.4) \times 10^{-4}$ \\
$\dot{P} b$ & $(1.7 \pm 2.6) \times 10^{-9}$ \\
$\dot{e}\left(\mathrm{~s}^{-1}\right)$ & $5(4) \times 10^{-15}$ \\
$\dot{x}$ & $6(1) \times 10^{-13}$ \\
\hline
\end{tabular}

Table 1. Timing parameters of PSR B1620-26. Position is relative to the JPL DE202 solar system ephemeris. Numbers in parentheses are uncertainties in the final digits quoted. NOTE: formal uncertainties are relative to model fit with above parameters. Covariances with unfit parameters (e.g., the fifth frequency derivative) may increase true uncertainties, particularly of $\dddot{f}$ and $\dddot{f}$.

Timing data were taken at the VLA and Green Bank (140-ft), using standard hardware and techniques. Observations at the VLA were made at $1.6 \mathrm{GHz}$; observations at Green Bank were made at a variety of frequencies between $390 \mathrm{MHz}$ and $1.6 \mathrm{GHz}$. The data span is 1988 March to 1995 December.

Timing parameters are reported in Table 1 , and in most cases represent an incremental improvement over previously published values. We report for the first time a value for $\dddot{f}$, but emphasize that despite its high significance in this fit, covariance with unmodeled parameters (e.g., $f$ ) may complicate its interpretation. An identical fit with a fifth order polynomial yields $\dddot{f}=$ 
$-1.5 \pm 2.2 \times 10^{-40} \mathrm{~s}^{-5}$ and $f=-1 \pm 6 \times 10^{-48} \mathrm{~s}^{-6}$ (the other frequency derivatives are unchanged).

The globular cluster proper motion in RA and Dec is $-9.7(7)$ and $-12.4(7)$ mas/yr (Cudworth \& Hansen 1993). This is about $2 \sigma$ from our timing proper motion, a difference that we do not believe is significant.

The optical proper motion has magnitude $\mu=2.42 \times 10^{-15} \mathrm{rads}^{-1}$. This will produce a change in the projected semi-major axis $x \equiv a_{1} \sin i$ of $\dot{x} / x=$ $\mu \sin j / \tan i$, where $i$ is the orbital inclination and $j$ is the angle between the proper motion vector and the line of nodes (neither $i$ nor $j$ is known). If the observed $\dot{x}$ is due to proper motion, then $\sin j<1$ implies $i<15^{\circ}$, which in turn implies that the companion must have mass $>1.6 M_{\odot}$, i.e., it is a neutron star or black hole, not a white dwarf. The nearly circular orbit is then problematic, but could be explained if the companion were captured in a three or four body interaction which involved a collision, followed by circularization during a Thorne-Zytkow phase or by a disk of material from a disrupted star. In our opinion, however, it is more likely that the observed $\dot{x}$ has, instead, some contribution from another source, such as precession.

At the most likely orbital inclination $i=60^{\circ}$, proper motion can account for only about $10 \%$ of the observed $\dot{x}$. A more attractive explanation is that $\dot{x}$ is caused by precession of the binary orbit in the gravitational field of the same third body that has been invoked to explain the large spin-frequency derivatives. It can be shown that this "planetary" precession yields $\dot{x} / x=G m_{2} F /\left(\Omega_{1} r_{2}^{3}\right)$, where $\Omega_{1}$ is the angular velocity of the inner orbit and $r_{2}$ is the distance to the second companion (assumed fixed). $F$ is a complex function of unknown angles, but has a median value for random orientations of 0.12 , a $50 \%$ range of $0.033-0.33$, and a $95 \%$ range of $0.0013-1.6$.

Assuming the inner binary has mass $M=m_{p}+m_{1}=1.7 M_{\odot}$ and a loweccentricity outer orbit, and ascribing the entire $\dot{x}$ to precession, we can write $F=\left(P_{2} / 3360 \mathrm{yr}\right)^{2}\left(1+M / m_{2}\right)$, where $P_{2}$ is the period of the outer orbit, and calculate $F$ for the three illustrative solutions of Rasio (1994). For $m_{2}=80_{\oplus}$ and $P_{2}=10 \mathrm{yr}, F=0.06$, while for $m_{2}=0.8 M_{\odot}$ and $P_{2}=120 \mathrm{yr}, F=0.004$, and for $m_{2}=1.4 M_{\odot}$ and $P_{2}=500 \mathrm{yr}, F=0.04$. We conclude that the measurement of $\dot{x}$ is consistent with the presence of a third companion, and indeed is a strong indication that the triple hypothesis is correct. However, by itself it is only a poor discriminator between stellar and planetary solutions. With more information from spin frequency derivatives and from other orbital perturbations such as $\dot{\omega}$ and $\dot{P}_{b}$, the measurement of $\dot{x}$ will help untangle the various unknown angles that characterize the system. A complete analysis of these orbital perturbation effects will be presented elsewhere (Joshi \& Rasio 1996).

\section{Orbital Parameters}

The best' test to confirm the triple nature of this system would be a Keplerian fit to the timing data spanning more than one orbit of the outer body. However, since the inferred orbital period $P_{2}$ is of order a century or more, we observe only a small portion of an orbit, effectively measuring successively higher order derivatives of the acceleration at a single point. If derivatives of the pulse frequency up to the fifth order were available, the system could in principle be 
solved completely (except for the usual inclination angle) for the orbital parameters and mass of the second companion. Here we use the values listed in Table 1 for frequency derivatives up to the fourth order to obtain a one-parameter family of solutions. While computing the orbital parameters of the second companion, we treat the inner binary as a single object of mass $M=1.7 M_{\odot}$. For fixed values of the eccentricity $e_{2}$ and the inclination $i_{2}$, we solve the non-linear system of equations for the frequency derivatives (using the Newton-Raphson method) for the mass $m_{2}$, the semi-major axis $a_{2}$, the angle of periastron $\omega_{2}$ and the longitude from pericenter $\lambda_{2}$ (Joshi \& Rasio 1996).

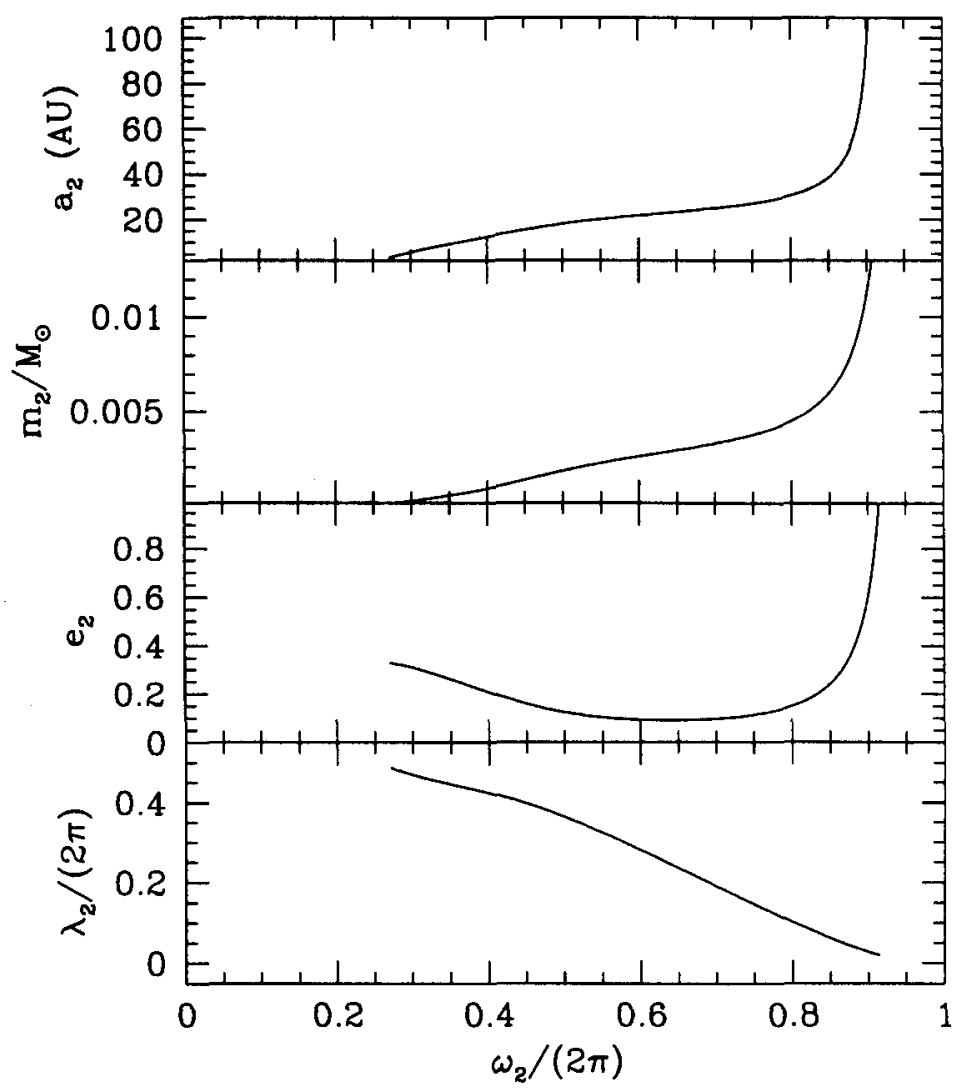

Figure 1. Our "standard solution," using the observed value of $\dddot{f}$.

Fig. 1 illustrates our "standard solution", obtained using the observed value of $\dddot{f}$. We see that there are no solutions for $e_{2} \lesssim 0.1$. Hence a nearly circular orbit is ruled out. For $0.1 \lesssim e_{2} \lesssim 0.3$ there are two solutions for each value of the eccentricity, and hence two possible values of $m_{2}$. In one solution, $m_{2}$ approaches zero as $e_{2}$ approaches $\approx 0.3$. However for $m_{2} \lesssim 10^{-5} M_{\odot}$ the triple configuration becomes dynamically unstable. In addition, we can rule out these 
solutions because they have very short orbital periods $\left(P_{2} \lesssim 5 \mathrm{yr}\right)$ and this would have been detected already in the timing data. For the other solutions, $m_{2}$ increases monotonically in the range $\sim 10^{-3}-10^{-2} M_{\odot}$ (Jupiter to browndwarf masses) as $e_{2}$ increases from $\sim 0.1$ to 1 .

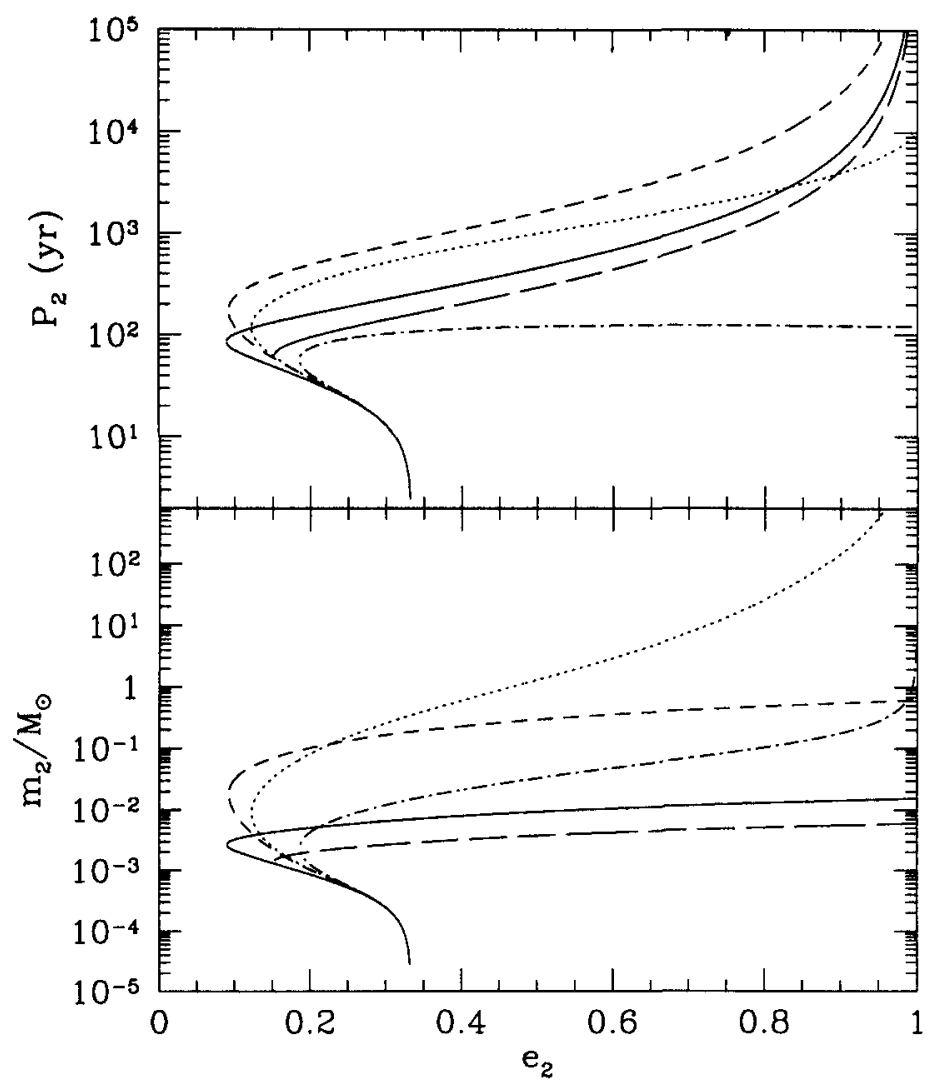

Figure 2. The solutions for $\dddot{f}=\dddot{f}_{m}$ (solid line), for $\dddot{f}=2.3 \dddot{f}_{m}$ (longdashed line), for $\dddot{f}=0.001 \dddot{f}_{m}$ (short-dashed line), for $\dddot{f}=-0.05 \dddot{f}_{m}$ (dotted line) and $\dddot{f}=-0.4 \dddot{f}_{m}$ (dot-dashed line). We assume here that the inclination angle $i_{2}=90^{\circ}$.

In Fig. 2 we show the results obtained on varying $\dddot{f}$ within a $4 \sigma$ error bar around the best fit value $\dddot{f}_{m}$ (given in Table 1). The results are shown for $\dddot{f} / \dddot{f}_{m}=1.0$ (as in Fig. 1, solid line), 2.3 (long-dashed line), 0.001 (shortdashed line), -0.05 (dotted line) and -0.4 (dot-dashed line). We find that stellar-mass solutions for $m_{2}$ are still possible if $-0.05 \lesssim \widetilde{f} / \dddot{f}_{m} \lesssim 0.001$. A second companion of stellar mass would provide a natural explanation for the eccentricity of the inner binary in terms of secular perturbations (Rasio 1994) 
and would also be consistent with a preliminary identification of an optical counterpart for the system (Bailyn et al. 1994).

\section{References}

Backer, D. C., Foster, R. S., \& Sallmen, S. 1993, Nature, 365, 817.

Bailyn, C. D., Rubinstein, E. P., Girard, T. M., Dinescu, D. I., Rasio, F. A. \& Yanny, B. 1994, ApJ, 433, L89.

Cudworth, K. M., \& Hansen, R. B. 1993, AJ, 105, 168.

Joshi, K. J., \& Rasio, F. A. 1996, In Preparation.

Lyne, A. G., Biggs, J. D., Brinklow, A., Ashworth, M., \& McKenna, J. 1988, Nature, $332,45$.

Michel, F. C. 1994, ApJ, 432, 239.

Rasio, F. A. 1994, ApJ, 427, L107.

Rasio, F. A., McMillan, S., \& Hut, P. 1995, ApJ, 438, L33.

Sigurdsson, S. 1995, ApJ, 452, 323.

Thorsett, S. E., Arzoumanian, Z., \& Taylor, J. H. 1993, ApJ, 412, L33. 